\title{
The Chip Design of A 32-b Logarithmic Number System
}

\author{
Sheng-Chieh Huang and Liang-Gee Chen ${ }^{*}$ \\ Department of Electrical Engineering \\ National Taiwan University \\ Taipei, Taiwan 10764, R.O.C. \\ (886)-(2)-3633174 ext 443 \\ Igchen@cc.ee.ntu.edu.tw
}

\begin{abstract}
To design a 32-bit logarithmic number system (LNS) processor, this paper presents two novel techniques : Digit-Partition (DP) to design $\log _{2}(1 . x)$ function and Iterative Difference by Linear Approximation (IDLA) to design $2^{a x}$ function. The experimental result reveals that the proposed design is more attractive than the previous researches in the LNS processor.
\end{abstract}

\section{MOTIVATION}

The central issue addressed by the previous LNS processors is the implementation of the functions $\log _{2}\left(1+2^{x}\right)$ and $\log _{2}\left(1-2^{x}\right)$. Conventionally, both functions $\log _{2}\left(1+2^{x}\right)$ and $\log _{2}\left(1-2^{x}\right)$ are implemented through methods of bit-serial [1], range reduction, table look-up and mathematical approximation [2][3]. However, they suffer either speed degradation or large chip-area cost when a high-precision design is desired. Only a few chip implementations of LNS arithmetic have been reported so far. An 20-bit LNS processor [2] is proposed to minimize this table complexity using a partitioned ROM and a PLA to achieve 12 bits fractional precision. Recently, a 30-bit LNS processor with 20 bits of fractional precision is reported in [3], which proposed a new algorithm for linear approximation using different-sized approximation intervals in each of a number of segments. To provide a higher precision, the hybrid number system (HNS) [4] has been introduced to perform logarithmic operations with 32-bit IEEE 754 single-precision floating-point data format. Anyway, the chip area may be too large to implement in a single chip.

In this paper, we design a logarithmic number system (LNS) processor, by two new techniques, Digit-Partition (DP) for $\log _{2}(1 . x)$ design and Interactive Difference by Linear Approximation (IDLA) for $2^{a x}$ design. The basic concept behind the design of $\log _{2}(1 . x)$ is that the wordlength of $x$ is divided into two parts to be implemented. Thus, ROM or PLA table can be reduced to

now is Research Consultant in AT\&T Bell Labs. Murry Hill. NJ

This work was supported by National Science Coumcil under Grant NSC 82-0404-E-002-218

\author{
Thou-Ho Chen \\ Department of Electronic Engineering \\ Nan-Tai College \\ Tainan, Taiwan 10764, R.O.C.
}

a reasonable size and this will make a high-precision design allowable. To design function $2^{a x}$ by IDLA, only adder, shifter, and a small PLA are required, unlike the previous designs which require ROM, and multiplier. Total hardware cost can be reduced, but it incurs a sacrifice of speed performance. To overcome this problem, the pipeline scheme is introduced. By using the proposed design of $\log _{2}(1 x)$ and $2^{a x}$, the logarithmic addition, $\log _{2}\left(1+2^{x}\right)$, and subtraction, $\log _{2}\left(1-2^{x}\right)$, can be performed with large number of bits.

\section{METHODOLOGY}

In the LNS, a number $x$ is represented by its sign and its logarithm to some base $b$. In this paper, we assume $b=2$. To represent a floating-point number, the representation of zero should be considered. For simplicity, zero is represented by using a zero flag $Z_{x}$. Therefore, the number $x$ is now represented by the triple $\left\langle S_{x}, Z_{x}, e_{x}>\right.$, and is shown as

$$
\mathrm{x}=\left(1-Z_{x}\right) \times(-1)^{s_{x}} \times 2^{e_{x}}
$$

Formally, $e_{x}$ is an $N$ bit fixed-point number with $I$ integer bits and $F$ fractional bits of precision, and $N=F+I$. Based on matching with IEEE single precision floating-point format, (1) can be rewritten as

$$
x=\left(1-Z_{x}\right) \times(-1)^{s_{x}} \times 2^{E_{x}+\log _{2}\left(M_{x}\right)}
$$

where $M_{x}$ is the 23-bit mantissa and $\log _{2}\left(M_{x}\right)$ has $F$ bits and $E_{x}$ has $I$ bits with a bias of execess- $\left(2^{i-1}-1\right)$.

\section{A. Primitive Arithmetic Operations}

Ignoring signs, those basic operations performed in the LNS will be described in the following

Multiplication :

$2^{c}=a \times b \Rightarrow c=E_{a}+\log _{2}\left(M_{d}\right)+E_{b}+\log _{2}\left(M_{b}\right)$

Division :

$2^{c}=a / b \Rightarrow c=E_{a}+\log _{2}\left(M_{a}\right)-\left(E_{b}+\log _{2}\left(M_{b}\right)\right)$

Addition :

$2^{c}=a+b \Rightarrow$

$c=\left(E_{a}+\log _{2}\left(M_{d}\right)\right)+\log _{2}\left(1+2^{E_{b}+\log _{2}\left(M_{b}\right)-\left(E_{a}+\log _{2}\left(M_{a}\right)\right.}\right)$

$=\left(E_{a}+\log _{2}\left(M_{d}\right)\right)+F_{+}\left(E_{b}+\log _{2}\left(M_{b}\right)-\left(E_{a}+\log _{2}\left(M_{d}\right)\right)\right)$

Subtraction :

$2^{c}=a-b \Rightarrow$ 
$c=\left(E_{a}+\log _{2}\left(M_{d}\right)\right)+\log _{2}\left(1-2^{E_{b}+\log _{2}\left(M_{b}\right)-\left(E_{a}+\log _{2}\left(M_{a}\right)\right.}\right)$

$=\left(E_{a}+\log _{2}\left(M_{d}\right)\right)+\mathrm{F}_{-}\left(E_{b}+\log _{2}\left(M_{b}\right)-\left(E_{a}+\log _{2}\left(M_{d}\right)\right)\right)$

(Here, $a<b \Rightarrow a-b<0$; if $(a-b)>0, F$. is invalid)

Exponent :

$2^{c}=a^{b} \Rightarrow c=\left(E_{a}+\log _{2}\left(M_{a}\right)\right) \times 2^{\left(E_{b}+\log _{2}\left(M_{b}\right)\right)}$

In the addition and subtraction, we define the auxiliary functions $F_{+}(z)$ and $F_{-}(z)$ as $\log _{2}\left(1+2^{z}\right)$ and $\log _{2}\left(1-2^{z}\right)$, respectively, where $z$ is an unsigned integer. Exponent operation in the floating-point number system consists of multiple multiplications. In LNS, only one multiplication is required. Besides, the multiplier can also support other functions in the LNS processor.

\section{B. Digit-Partition (DP)}

The algorithm of digit-partition is composed of three steps and described as follows:

$$
\begin{array}{ll}
\text { Step 1: Digitalization } & \\
& \log _{2}(1 . x)=0 . y \\
\Rightarrow \quad & \log _{2}\left(1 . x_{0} x_{1} x_{2} \ldots x_{12}\right)=0 . y_{0} y_{1} y_{2} \ldots y_{11} \\
\Rightarrow \quad \log _{2}\left(1 . x_{0} x_{1} x_{2} \ldots x_{22}\right)=0 . y_{0} y_{2} y_{2} \ldots y_{22}
\end{array}
$$$$
\text { DP Algorithm }
$$

In the point of designing, it is intractable to implement equation (4) directly with ROM or PLA due to its large size, about $2^{23}$ words. Based on the experimental results, if both values of $x_{0} x_{1} x_{2} \ldots x_{12}$ in (3) and (4) are identical, both values of $y_{0} y_{1} y_{2} \ldots y_{I I}$ are also the same. In other words, $\log _{2}\left(0.00 \ldots x_{13} x_{18} x_{15} \ldots x_{22}\right)$ only dominates $0.00 \ldots 0 y_{13} y_{13} y_{14} \ldots y_{22}$ value, not including $0 . y_{0} y_{1} y_{2} \ldots y_{1 I}$. Step 2 : Dividing

$$
\begin{aligned}
& 1 . x_{0} x_{1} x_{2} \ldots x_{22}=2^{0 . y_{0} y_{1} y_{2} \ldots y_{22}} \\
& =\left(2^{0 . y_{0} y_{1} y_{2} \ldots y_{11}}\right)\left(2^{0.000 \ldots 00 y_{12} y_{13} \ldots y_{22}}\right) \\
\Rightarrow & \left(1 . x_{0} x_{1} x_{2} \ldots x_{22}\right) *\left(2^{-0 . y_{0} y_{2} y_{2} \ldots y_{11}}\right)=2^{0.000 \ldots 00 y_{12} y_{13} \ldots y_{22}} \\
\Rightarrow & \left(1 . x_{0} x_{1} x_{2} \ldots x_{12}\right)^{*}\left(2^{-0 . y_{0} y_{1} y_{2} \ldots y_{11}}\right)+\left(0.000 \ldots 0 x_{13} x_{14} x_{15} \ldots x_{22}\right)^{*} \\
& \left(2^{-0 . y_{0} y_{1} y_{2} \ldots y_{11}}\right)=2^{0.000 \ldots 0 y_{12} y_{13} \ldots y_{22}}
\end{aligned}
$$

In equation (6), $\left(1 . x_{0} x_{1} x_{2} \ldots x_{12}\right)^{*}\left(2^{-0 . y_{0} y_{1} y_{2} \ldots y_{11}}\right)$ term can be obtained by using a 13-bit PLA with input $x_{0} x_{1} x_{2} \ldots x_{12}$. Another term, $\left(0.000 \ldots 0 x_{13} x_{18} x_{15} \ldots x_{22}\right)^{*}\left(2^{-0 . y_{0} y_{1} y_{2} \ldots y_{11}}\right)$, can be derived by the $\log _{2}(x)$ and $2^{x}$ function as follows:

$$
\begin{aligned}
& \left(0.000 \ldots 0 x_{13} x_{14} x_{15} \ldots x_{22}\right) *\left(2^{-0 . y_{0} y_{1} y_{2} \ldots y_{11}}\right)
\end{aligned}
$$

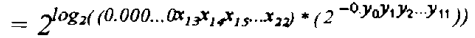

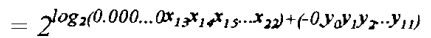

$$
\begin{aligned}
& =2^{\log _{2}\left(0 . x_{13} x_{1}, x_{15} \ldots x_{22}\right)+(-13)+\left(-0 . y_{0} y_{2} y_{2} \ldots y_{11}\right)}
\end{aligned}
$$

By using equation (6), (7) can be rewritten as

$$
\left(1 . x_{0} x_{2} x_{2} \ldots x_{12}\right) *\left(2^{-0 . y_{0} y_{1} y_{2} \cdot y_{11}}\right)+
$$$$
2^{\log _{2}\left(0 . x_{13} x_{1} x_{15} \ldots x_{22}\right)+(-13)+\left(-0 y_{0} y_{1} y_{3} \cdots y_{11}\right)}=2^{0.000 \ldots 00 y_{12} y_{13} \cdots y_{23}}
$$

$\Rightarrow\left(1 . x_{0} x_{1} x_{2} \ldots x_{12}\right) *\left(2^{-0 y_{0} y_{1} y_{2} \cdots y_{11}}\right)+$

$\left(2^{-13}\right)\left(2^{\log _{20}\left(0 . x_{13} x_{13} x_{15} \ldots x_{22}\right)+\left(-0 y_{0} y_{1} y_{2} \ldots y_{13}\right)}\right)=2^{0.000 \ldots .00 y_{3} y_{13} \ldots y_{22}}$

Step 3 : Group-Merging

In equation (8), $2^{-13}$ means the 13 bits right shifting, and $2^{\log _{2}\left(0, x_{13} x_{1} x_{15} \ldots x_{22}\right\}+\left\{-0 y_{0} y_{1} y_{2}, y_{11}\right)}$ is transformed into another representation $2^{-I . F}$ where $2^{-I}$ can be realized by the $I$ bits right shifting and $2^{-a F}$ can be realized by function unit $\left(2^{a x}\right)$ as described in the next section. To derive $2^{0.000 \ldots .00 y_{1} y_{13} . . y_{22}}$ with small chip size, the mapping ratio [2] and DGPLA [5] techniques are introduced.

\section{Iterative Difference by Linear Approximation (IDLA)}

In the design of commercial product, $2^{a_{x}}$ is computed by using the polynomial approximation and the following function.

$$
2^{a x}=\left(2^{a x / 2}+1\right)\left(2^{a x / 2}-1\right)+1=\left(\left(2^{a x / 2}-1\right)+2\right)\left(2^{a x / 2}-1\right)+1
$$

By using the above equation, it can reduce the parameter range about half. Although, an additional multiplier is required. Besides, using the polynomial approximation will degrade the speed performance due to iterative multiply operations.

To overcome the above drawbacks, we propose a novel algorithm, called Iterative Difference by Linear Approximation (IDLA), to compute $2^{a x}$. The basic concept behind this algorithm is that the function $2^{a x}$ can be obtained approximately through iterative linear approximations. The slope can be tuned to function $2^{\prime}$, where $I$ is integer. Thus, only the adder and shifter are required to implement $2^{a x}$. If the difference $d_{i}(x)$ between $2^{0 . x}$ and linear function $y_{i}$, i.e. $d_{i}(x)=2^{0 . x}-y_{i}$ is more than the permissible error, the next linear approximation operation should be presented as $d_{i j}(x)=d_{i}(x)-y_{j}$. Such iterative linear approximations must be continued until the precision requirement is achieved. The IDLA algorithm can be described as follows :

$$
\text { IDLA Algorithm }
$$

step 0: Let $f(x)=2^{a x}, 0 \leq 0 . x<1$

step 1: Splitting $f(x)$ into $2^{n_{0}}$ subsections, and $i$-th subsection is denoted as $f_{i}(x)$, as shown in Fig. 1(a), and $0 \leq i \leq 2^{n_{0}}-1$ where $n_{0}$ is a positive integer.

step 2 : Using linear approximation to obtain value of $f(x)$ at each subsection $f_{i}(x)$.

Let the difference between $f_{i}(x)$ and its linear approximate function $y_{i}=m_{i} x+k_{i}$ to be $d_{i}(x)=$ $f_{i}(x)-y_{i}$, and $d_{i, \max }$ is the maximum value of $d_{i}(x)$

step 3: $\operatorname{IF}\left|d_{i, \text { max }}\right|>\operatorname{Er}$ (i.e. the permissible error) THEN splitting $f_{i}(x)$ into $2^{n_{i}}$ subsections, each subsection is denoted as $f_{i j}(x), 0 \leq j \leq 2^{n_{i}}-1$

ELSE goto step 6

step 4: Using linear approximation to obtain value of $d_{j}(x)$ at each subsection $f_{i j}(x)$

Let $y_{j}=m_{f} x+k_{j}, 0 \leq j \leq 2^{n_{i}}-1$

$d_{i j}(x)=f_{i j}(x)-y_{j}, d_{i j, \text { max }}$ is the maximum value of $d_{i j}(x)$

step $5: \operatorname{IF}\left|d_{i j, \max }\right|>\operatorname{Er}$ THEN

splitting $d_{i j}(x)$ into $2^{n_{i}}$ subsections, and then goto step 4 to judge each subsection again

ELSE

goto step 6 
step 6: Return $f(x)=\Sigma\left(\left(m_{f} x+k\right)\right.$ of each iteration $)$

In the above algorithm, the permissible error $E_{r}$ is defined according to the precision requirement. For the purpose of using only the adder and shifter to implement $2^{0 . x}, m_{i}$ is selected as $2^{-I}$ or $1-2^{-I}$, where $I$ is an integer, if $m_{i}$ approximates 0 or 1 , respectively. The selection of $k_{\mathrm{i}}$ depends on the following two cases of $f_{i}(x)$ :

Case 1: As shown in Fig. 1(b).

$L_{1}: y=m_{1} x+A, L_{1}$ passes point $P$

$L_{2}: y=m_{1} x+k_{0}, L_{2}$ passes tangent point $Q$ of $f_{i}(x)$ and is parallel with $L_{L}$ the desired line is $y=m_{f} x+k_{i}, k_{i}=\left(A+k_{o}\right) / 2$, where $L_{1}, L_{2}$ and the desired line are all parallel Case 2: As shown in Fig. 1(c).

$L_{1}: y=m_{f} x+A, L_{1}$ passes point $P$

$L_{2}: y=m_{1} x+B, L_{2}$ passes point $Q$

the desired line is $y=m_{i} x+k_{i}, k_{i}=(A+B) / 2$,

where $L_{1}, L_{2}$ and the desired line are all parallel

D. $\log _{2}\left(1+2^{x}\right)$ and $\log _{2}\left(1-2^{x}\right)$

If $x$ is normalized, $\log _{2}(x)$ can be replaced by $\log _{2}(1 . x)$ in order to append one bit of precision and $2^{x}$ is replaced by $2^{0 . x}$. Thus we firstly build circuits of function $\log _{2}(1 . x)$ by DP and $2^{0 x}$ by IDLA and then both are utilized to perform $\log _{2}\left(1+2^{x}\right)$ and $\log _{2}\left(1-2^{x}\right)$

The derivation of $\log _{2}\left(1+2^{x}\right)$ algorithm is composed of three parts. Firstly, under satisfying the precision requirement, the parameter range can be reduced to a smaller interval. In this interval, we utilize the essential zero and linear equivalent methods to derive the lower bound threshold and upper bound threshold, respectively. Secondly, $1+2^{x}$ is transformed to another form, i.e., $1+2^{x}=$ $1+2^{I . F}=1+\left(2^{J}\right)\left(2^{a . F}\right)$ when $x \geq 0$, and $1+\left(2^{-J}\right)\left(2^{-\alpha . F}\right)$ when $\mathrm{x}<$ $0.2^{I}$ and $2^{I}$ will be implemented by shifter, and $2^{a . F}$ and $2^{-O F}$ will be realized as described in the above subsection. Nevertheless, when $x \geq 0$, the range of $1+2^{x}$ will become very large after shifting $2^{a F}$ left by $I$ bits. Finally, to reducing the range, $\log _{2}\left(1+\left(2^{I}\right)\left(2^{O . F}\right)\right)$ is written as $I+$ $\log _{2}\left(2^{-I}+2^{O . F}\right)$ and now $2^{-I}$ is realized by adding one to the $i$-th position of $2^{a F}$. When $\mathrm{x}<0, \log _{2}\left(1+\left(2^{-1}\right)\left(2^{-\alpha . F}\right)\right)$ can be implemented directly by the $\log _{2}(1 . x)$ function since it has a small range, i.e., $1.5<1+\left(2^{-I}\right)\left(2^{-a . F}\right) \leq 2$. For $\log _{2}\left(1-2^{x}\right)$, there is the same discussion as that of $\log _{2}\left(1+2^{x}\right)$.

\section{CHIP IMPLEMENTATION}

For brevity, the chip design of 32-bit LNS processor can be decomposed into four parts : $\log _{2}(1 . x), 2^{0 . x}, \log _{2}\left(1+2^{x}\right)$, and $\log _{2}\left(1-2^{x}\right)$. For $\log _{2}(1 x)$, the core hardware consists of one $8192 \times 12 \mathrm{~b}$ ROM, three PLAs (sizes of PLA1, PLA2 and PLA 3 are $15850 \mathrm{~b}, 11400 \mathrm{~b}$ and $14380 \mathrm{~b}$ ), two adders (adder1: 13-b and adder2: 11-b) and one 24-b shifter as shown in Fig. 2. Fig. 3 shows the block diagram of $2^{a x}$ design and the core hardware consists of two PLAs (size of slope PLA is $9536 \mathrm{~b}$ and size of offset PLA is $117435 \mathrm{~b}$ ), one 24-b shifter and four 23-b adders/subtractors. The core hardware of $\log _{2}\left(1+2^{x}\right)$, and $\log _{2}\left(1-2^{x}\right)$ consists of functional units $2^{\alpha F}$ and $2^{-a F}$, as described in Fig. 4 and Fig. 5 , respectively.

The chip of 32-bit LNS processor is completed by using the structural silicon compiler in Genesil system with TSMC $0.8 \mu \mathrm{m}$ technology. The chip size is $90 \mathrm{~mm}^{2}$ and it can be operated at $25 \mathrm{MHz}$. Fig. 6 shows layout of the chip.

The proposed 32-bit LNS processor will be compared with three previous LNS processors in terms of the bit number, look-up table size, speed, and chip area, as illustrated in Table I.

\section{CONCLUSIONS}

This paper proposes the digit-partition (DP) technique to design $\log _{2}(1 . x)$ function and Iterative Difference by Linear Approximation (IDLA) to design $2^{a x}$ function with high precision. By DP, $\log _{2}(1 x)$ with large bit number of $x$ can be achieved with moderate hardware cost. By IDLA, we can design $2^{a x}$ function with only adder and shifter. Thus, the logarithmic addition $\log _{3}\left(1+2^{x}\right)$ and subtraction $\log _{2}\left(1-2^{x}\right)$ can be realized with the proposed $\log _{2}(1 . x)$ and $2^{a x}$, to achieve a high-precision capability. Based on the proposed 32-bit LNS processor which satisfies the IEEE 754 standard, we hope that the digit partition and IDLA technique will be further applied to the higher precision number system while showing cost-effective and high-precision design in the future.

\section{REFERENCES}

[1] H. Y. Lo, J. L. Chen, "A Hardwired Generalized Algorithm for Generating the Logarithm Base-k by Iteration," IEEE Trans. Comput., vol. C-36, pp. 1363-1367, Nov. 1987

[2] F. J. Taylor, R. Gill, J. Joseph, J. Radke, "A 20 Bit Logarithmic Number System Processor," IEEE Trans. Comput., vol. 37, pp. 190-200, Feb. 1988.

[3] Lawrence K.Yu, David M. Lewis, "A 30-b Integrated Logarithmic Number System Processor," IEEE J. Solid-State Circuits, vol. 26, pp. 1433-1440, Oct. 1991.

[4] F. S. Lai, "The Architecture and Analysis of a Hybrid Number System Processor," IEEE Sym. on Circuit and System, pp. 803-806, 1992.

[5] H. Y. Lo, Y. Aoki, "Generation of a Precise Binary Logarithm with Difference Grouping Programmable Logic Array," IEEE Trans. Comput., vol. C-34, pp. 681-691, Aug. 1985. 


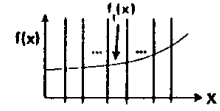

(b)
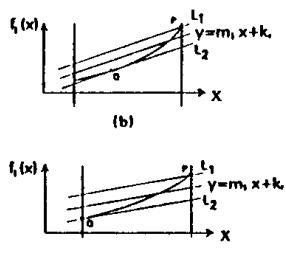

(c)

Fig. 1. Itcrative Dirference by Linear Approximation (IDLA).

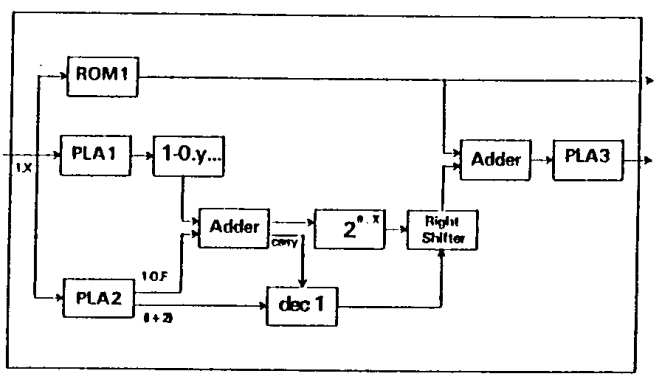

Fig. 2. The block diagram of $\log _{2}(1 . x)$ design.

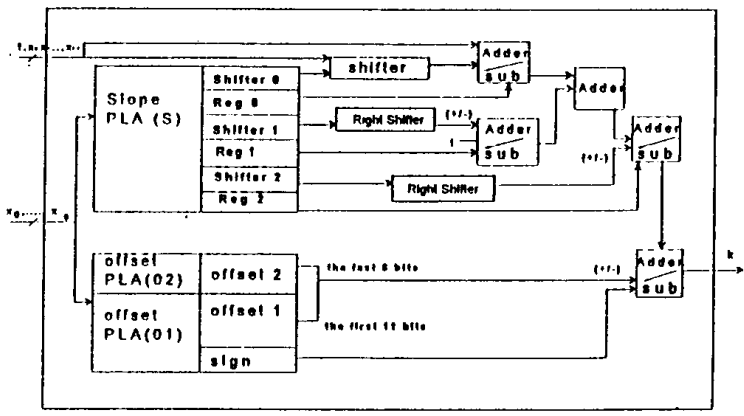

Fig. 3. The block diagram of $2^{0, x}$ Jesign.
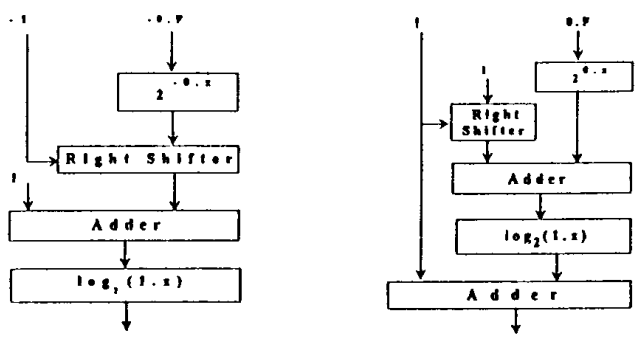

(i) $x<0$

(ii) $x \geq 0$
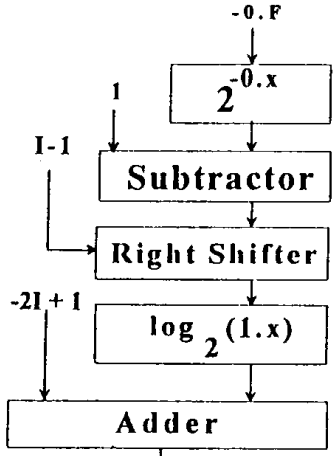

Fig. 5. The block diagram of $\log _{2}\left(1-2^{x}\right)$ design.

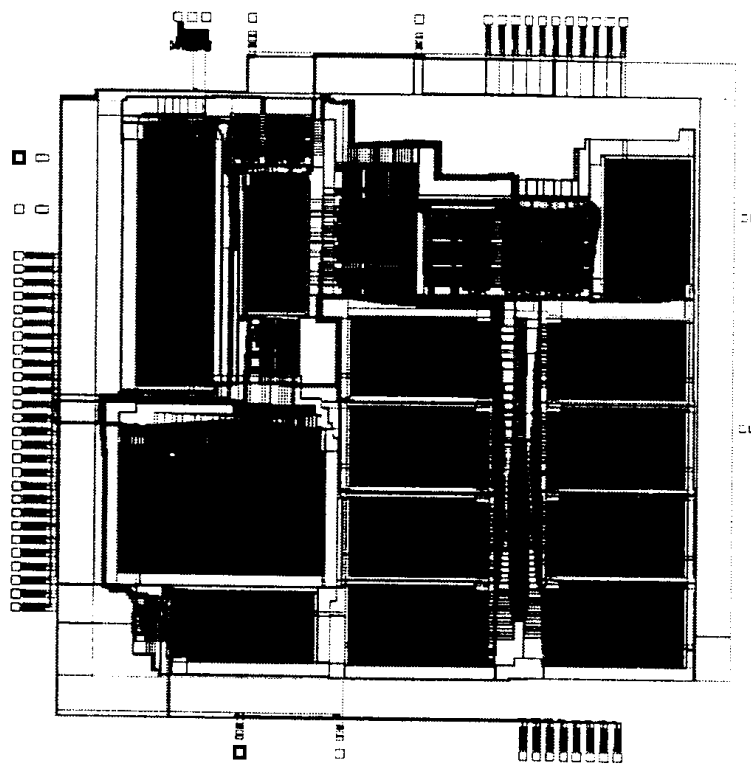

Fig. 6. Layout of 32-bit LNS processor.

\begin{tabular}{|c|c|c|c|c|c|c|c|c|}
\hline method & $\begin{array}{l}\begin{array}{l}\text { memory } \\
\text { size(K) }\end{array} \\
\end{array}$ & $\log _{2}(x)$ & $2^{x}$ & $\log _{2}\left(1+2^{x}\right)$ & $\log _{\frac{2}{2}}\left(1-2^{x}\right)$ & speed & \begin{tabular}{|l|} 
chip \\
area
\end{tabular} & technology \\
\hline $20-b x t[2]$ & $202 \mathrm{~K}$ & $48 \mathrm{X}$ & - & $7 \pi \mathrm{x}$ & $n \mathrm{k}$ & . & $40 \operatorname{son}^{2}$ & ISL \\
\hline 30-bit [3] & $257 \mathrm{~K}$ & $18 \mathrm{~K}$ & $28 \mathrm{~K}$ & $21 \mathrm{~K}$ & $172 x$ & $56 \mathrm{MHz}$ & $100 \mathrm{~mm}^{2}$ & $3 \mu \mathrm{m} \mathrm{CMOS}$ \\
\hline HNS [4] & $290 \mathrm{~K}$ & $135 \mathrm{~K}$ & $137 \mathrm{~K}$ & \multirow{2}{*}{\multicolumn{2}{|c|}{ combinabostal circair }} & • & - & - \\
\hline $32-b i t *$ & $266 \mathrm{~K}$ & $139 \mathrm{~K}$ & $126 \mathrm{~K}$ & & & $25 \mathrm{MHz}$ & $90 \mathrm{~mm}^{2}$ & $0.8 \mathrm{~m} \mathrm{CMOS}$ \\
\hline
\end{tabular}

Fig. 4. The block diagram of $\log _{2}\left(1+2^{x}\right)$ design. 